\title{
Enacting emotional interpretations with feeling
}

\author{
Giovanna Colombetti and Evan Thompson \\ Department of Philosophy, York University, Toronto, Ontario M3J 1P3 \\ Canada \\ colombet@yorku.ca http://www.arts.yorku.ca/phil/colombet \\ evant@yorku.ca http://www.yorku.ca/evant
}

\begin{abstract}
This commentary makes three points: (1) There may be no clear-cut distinction between emotion and appraisal "constituents" at neural and psychological levels. (2) The microdevelopment of an emotional interpretation contains a complex microdevelopment of affect. (3) Neurophenomenology is a promising research program for testing Lewis's hypotheses about the neurodynamics of emotion-appraisal amalgams.
\end{abstract}

One way to think about Lewis's portrayal of appraisal-emotion interactions is by comparison with dynamic sensorimotor approaches to perception and action (Varela et al. 1991; O’Regan \& Noë 2001; Hurley \& Noë 2003). According to these approaches, perception is as much a motor process as a sensory one. At the neural level, there is "common coding" of sensory and motor processes (e.g., Prinz 1997; Rizzolatti et al. 1997). At the psychological level, action and perception are not simply instrumentally related, as means-to-end, but are constitutively interdependent (Hurley 1998). These and other findings can be described by saying that perception is enactive: it is a kind of action (Varela et al. 1991; Noë 2004).

Lewis's target article can be read as presenting a logically analogous way of thinking about cognition and emotion. At the neural level, brain systems traditionally seen as subserving separate functions of appraisal and emotion are inextricably interconnected. Hence "appraisal" and "emotion" cannot be mapped onto separate brain systems. At the psychological level, appraisal and emotion are constitutively

interdependent: one is not a mere means to the other (as in the idea that an appraisal is a means to the having of an emotion, and vice versa); rather, they form an integrated and self-organizing emotion-appraisal state, an "emotional interpretation."

Although the target article ends with this kind of account (see in particular the last two paragraphs), the beginning seems more traditional. Lewis individuates emotion components and appraisal components, and maps them onto distinct brain systems. Emotion and appraisal have some components in common (attentional systems in particular), and their components are highly distributed. Nevertheless, some brain systems and functions are only emotional and do not belong to appraisal (e.g., arousal and feeling), and some belong only to appraisal and not emotion (e.g., planning). Some brain systems constitute either emotion or appraisal (or both), and some merely interact with one or the other. 
Lewis presents the emotion/appraisal distinction as an initial heuristic for looking at brain processes. We agree that one must start somewhere. Yet we wonder how much conceptual change Lewis thinks his view of a deeply integrated and dynamic brain implies for the psychological taxonomy with which he began. Consider that his dynamic approach is consistent with other, different views of the relationship between emotion and appraisal. Scherer (2000), for instance, also believes that appraisal and emotion components interact in a way best explained in dynamical terms, but he sees appraisal as a component of emotion. Freeman (2000) thinks that emotion is an endogenously generated (mainly limbic) dynamic activity pattern that mediates sensorimotor loops by providing different degrees of salience to events. According to this view, emotion is a constitutive element of any cognitive process, so that there is no theoretical room for nonemotional appraisals. This neurodynamic account is consistent with phenomenological accounts, according to which perception and evaluation are emotive and valenced (Varela \& Depraz 2000; Thompson, forthcoming).

Although we cannot argue the case here, and although we realize this view is outside the mainstream of emotion theory with which Lewis is concerned to communicate, we nevertheless believe that it may ultimately prove unproductive even to try to differentiate distinct "appraisal constituents" and "emotion constituents," which then "interact" in the formation of an emotional interpretation. Rather, we suspect that there may be no appraisal constituent that is not also an emotion constituent, and vice versa. For example, Lewis describes feeling as a component of emotion, but not appraisal. When an emotional interpretation starts to emerge, feeling plays an important role in modulating appraisals, but it is not itself an appraisal constituent (see what happens to Mr. Smart in the target article). Yet there is a "feeling of appraisal," and appraisal can be seen as constitutive of emotion experience (Frijda 1986). Hence categorizing feeling as an emotion constituent but not also an appraisal constituent seems limited.

Although feeling plays an important motivational role in Lewis's model, he does not explore the phenomenology of affect (the experiential aspect of emotion) in relation to the emergence of an emotional interpretation. Yet the microdevelopment of an emotional interpretation contains within it a complex microdevelopment of affect. Consider a momentary emotional interpretation, such as seeing a stranger's angry face glaring at you as you walk by. One can point to a number of concurrent components of affect in such an episode (see also Watt 1998):

A precipitating event or trigger, which can be perceptual or imaginary, or both. This component corresponds to the trigger phase in Lewis's model.

An emergence of affective salience, involving a sense of the precipitating event's significance. The emotion/appraisal processes leading to the emergence of this affective salience could reflect the kind of self-amplification and self-stablization processes Lewis describes.

A hedonic tone, along a pleasant/unpleasant polarity. 
A motor embodiment, in the form of facial and posture changes, and differential action tendencies or global intentions for acting on the world.

A visceral-interoceptive embodiment, in the form of complex autonomicphysiological changes (to cardiopulmonary parameters, skin conductance, muscle tone, and endocrine and immune system activities).

Neuroscientists have recently emphasized the link between affect and "core consciousness" or the feeling of self (Panksepp 1998; Damasio 1999), an idea also central to phenomenological philosophy. These two streams of neuroscience and phenomenology intersect in the research program of "neurophenomenology" (Varela 1996; Lutz \& Thompson 2003; Thompson et al., in press). Lewis's (2000) model of emotion-appraisal amalgams at multiple time-scales, together with a richer account of the role of affect in the development of emotional interpretations, can both inform neurophenomenological research on emotion, and also benefit from its rigorous way of linking first-person phenomenological reports and neurodynamical studies of large-scale integration (see Lutz et al. 2002). In particular, neurophenomenology provides a promising research program for exploring and testing Lewis's hypotheses about synchronous/asynchronous interactions across gamma and theta frequency bands in corticolimbic systems (see also Friston 2000; Varela et al. 2001).

\section{References}

Damasio, A. R. (1999) The feeling of what happens. Harcourt Brace. [GC]

Freeman, W. J. (2000) Emotion is essential to all intentional behaviors. In:. Emotion, development, and self-organization, ed. M. D. Lewis \& I. Granic. Cambridge University Press. [GC]

Frijda, N. H. (1986) The emotions. Cambridge University Press. [GC]

Friston, K. J[PC1]. (2000) The labile brain: II. Transients, complexity, and selection. Philosophical Transactions of the Royal Society of London B. 355:237-52. [GC]

Hurley, S. L. (1998) Consciousness in action. Harvard University Press. [GC]

Hurley, S. L. \& Noë, A. (2003) Neural plasticity and consciousness. Biology and Philosophy 18:131-68. [GC]

Lewis, M. D. (2000) Emotional self-organization at three time scales. In: Emotion, development, and self-organization, ed. M. D. Lewis \& I. Granic. Cambridge University Press. [GC]

Lutz, A., Lachaux, J.-P., Martinerie, J. \& Varela, F. J. (2002) Guiding the study of brain dynamics by using first-person data: Synchrony patterns correlate with ongoing 
conscious states during a simple visual task. Proceedings of the National Academy of Sciences USA 99:1586-91. [GC]

Lutz, A \& Thompson, E. (2003) Neurophenomenology: Integrating subjective experience and brain dynamics in the neuroscience of consciousness. Journal of Consciousness Studies 10:31-52. [GC]

Noë $(2004[\mathrm{PC} 2])$

O’Regan, K. J. \& Noë, A. (2001) A sensorimotor account of vision and visual consciousness. Behavioral and Brain Sciences 24:883-917. [GC]

Panksepp, J. (1998) The periconscious substrates of consciousness: Affective states and the evolutionary origins of self. Journal of Consciousness Studies 5:566-82.

[GC]

Prinz, W. (1997) Perception and action planning. European Journal of Cognitive Psychology 9:129-54. [GC]

Rizzolatti, G., Fadiga, L., Fogassi, L. \& Gallese, V. (1997) The space around us. Science 277:190-91. [GC]

Scherer, K. R. (2000) Emotions as episodes of subsystem synchronization driven by nonlinear appraisal processes. In: Emotion, development, and self-organization, ed. M. D. Lewis \& I. Granic. Cambridge University Press. [GC]

Thompson, E. (forthcoming[PC3]) Radical embodiment: The lived $[\mathrm{PC} 4]$ body in biology, human experience, and the sciences of mind. Harvard University Press. [GC]

Thompson, E., Lutz, A. \& Cosmelli, D. (in press[PC5]) Neurophenomenology: An introduction for neurophilosophers. Forthcoming in:. Cognition and the brain: The philosophy and neuroscience movement, ed. A. Brook \& K. Akins. Cambridge University Press. [GC]

Varela, F. J. (1996) Neurophenomenology: A methodological remedy for the hard problem. Journal of Consciousness Studies 3:330-50. [GC]

Varela, F. J. \& Depraz, N. (2000) At the source of time: Valence and the constitutional dynamics of affect. Arob@se. Journal de lettre et de sciences humain 4(1-2). http://www.arobase.to. Also published in S. Gallagher and S. Watson (eds), Ipseity and alterity: Interdisciplinary approaches to intersubjectivity. Presses Universitaires de Rouen, in press[PC6]. [GC]

Varela et al. (2001[PC7])

Varela, F., Thompson, E. \& Rosch, E. (1991) The embodied mind. MIT Press. [GC] 
Watt, D. F. (1998) Emotion and consciousness: Implications of affective neuroscience for extended reticular thalamic activating system theories of consciousness.

Electronic publication of the Association for the Scientific Study of

Consciousness. Available at: http://server.philvt.edu/assc/watt/default.htm. [GC] 